\title{
Lie Symmetry Reductions, Exact Solutions and Conservation Laws of the Third Order Variant Boussinesq System
}

\author{
E. YAŞAR* AND İ.B. GiRESUnLU \\ Department of Mathematics, Faculty of Arts and Sciences, Uludag University, 16059, Bursa, Turkey
}

(Received March 27, 2015)

\begin{abstract}
The Lie group method is applied to the third order variant Boussinesq system, which arises in the modelling of the water waves. The symmetry reductions and invariant solutions are obtained with respect to Lie point symmetry generators of the underlying system. In addition, we derive conservation laws of the variant Boussinesq system.

DOI: 10.12693/APhysPolA.128.RK.128.3.243-252

PACS/topics: 02.30.Ik, 02.30.Jr, 47.35.Fg
\end{abstract}

\section{Introduction}

The research area of nonlinear partial differential equations (NLPDEs) has been very active for the past few decades. The study of the exact solutions of a nonlinear evolution equation (NLEE) plays an important role to understand the nonlinear physical phenomena which are described by these equations. In recent years several powerful and efficient methods have been developed for finding analytic solutions of NLEEs. Some of the most important methods found in the literature include the inverse scattering, the Hirota bilinear method, the Darboux transformation method, $G^{\prime} / G$ expansion method, homogeneous balance method, Adomian decomposition method, the functional variable method, the extended tanh function method, etc. [1-7].

One such NLEE is the third order variant Boussinesq system and is given by

$$
v_{t}+v u_{x}+u v_{x}+u_{x x x}=0, \quad u_{t}+v_{x}+u u_{x}=0 .
$$

This system was introduced as a model for water waves $[8,9]$ where $u$ is the velocity and $v$ the total depth, and the subscripts denote partial derivatives. In Ref. [10], the solitary wave solutions of the variant Boussinesq equations are obtained by using a homogeneous balance method. In Ref. [8], the authors constructed the soliton solutions, rational solutions, triangular periodic solutions, Jacobi and Weierstrass doubly periodic wave solutions using the extended tanh method. In Refs. [9] and [11], the conservation laws for the variant constant and variable coefficients Boussinesq system are derived by the Noether approach.

Since the end of the 19th century, the symmetry study which laid the foundations of Lie plays an important role in almost all the scientific fields. As mentioned in [12], theory of Lie groups for obtaining the group invariant solutions to NLPDEs is widely recognized as one of the most powerful methods. We observe a plenty of books and survey articles about Lie groups method [13, 14].

*corresponding author; e-mail: eyasar@uludag.edu.tr
Moreover, the method systematically applied to differential equations arising in a different fields $[15,16]$.

It is well known that, in all areas of physics, conservation laws are essential since they allow us to draw conclusions of a physical system under study in an efficient way [17]. They are used for analysis, in particular, existence, uniqueness, and stability analysis and construction of numerical schemes. Moreover, conservation laws are used in obtaining the new nonlocal symmetries, nonlocal conservation laws and linearization [18]. A variety of powerful methods, have been used to investigate conservation laws of PDEs [9, 11, 14, 19-27].

The outline of the paper is as follows. In Sect. 2, the Lie symmetry methods along with the simplest equation method are employed to obtain exact solutions of (1). Then in Sect. 3, we construct conservation laws for (1) using the multiplier method. Finally, in Sect. 4 concluding remarks are presented.

\section{Symmetry reductions and exact solutions of Eq. (1)}

In this section, we present the notations and some of the definitions below. For the details see e.g., $[9,14$, 23-25]. The symmetry group of the variant Boussinesq system (1) will be generated by the vector field of the form

$$
\begin{gathered}
X=\xi(t, x, u, v) \frac{\partial}{\partial x}+\tau(t, x, u, v) \frac{\partial}{\partial t} \\
+\eta(t, x, u, v) \frac{\partial}{\partial u}+\phi(t, x, u, v) \frac{\partial}{\partial v} .
\end{gathered}
$$

Applying the third order prolongation $\operatorname{pr}^{(3)} X$ to (1) results in an overdetermined system of linear partial differential equations. The general solution of the overdetermined system of linear partial differential equations is given by

$$
\begin{aligned}
& \tau(t, x, u, v)=-c_{1} t+c_{2}, \xi(t, x, u, v)=c_{3} t-\frac{1}{2} c_{1} x+c_{4}, \\
& \eta(t, x, u, v)=\frac{1}{2} c_{1} u+c_{3}, \phi(t, x, u, v)=c_{1} v .
\end{aligned}
$$

The above general solution contains four arbitrary constants. Hence the infinitesimal symmetries of (1) form the four-dimensional Lie algebra spanned by the following linearly independent operators (see also [26]): 


$$
\begin{aligned}
& X_{1}=\frac{\partial}{\partial t}, \quad X_{2}=\frac{\partial}{\partial x}, \quad X_{3}=\frac{\partial}{\partial u}+t \frac{\partial}{\partial x}, \\
& X_{4}=-\frac{1}{2} x \frac{\partial}{\partial x}-t \frac{\partial}{\partial t}+\frac{1}{2} u \frac{\partial}{\partial u}+v \frac{\partial}{\partial v} .
\end{aligned}
$$

In order to obtain symmetry reductions and exact solutions, one has to solve the associated Lagrange equations

$$
\frac{\mathrm{d} t}{\tau(t, x, u, v)}=\frac{\mathrm{d} x}{\xi(t, x, u, v)}=\frac{\mathrm{d} u}{\eta(t, x, u, v)}=\frac{\mathrm{d} v}{\phi(t, x, u, v)} .
$$

We consider the following cases:

Case 1. $c_{1}=\alpha, c_{3}=1, c_{2}=c_{4}=0$.

The symmetry $\alpha X_{1}+X_{3}$ gives rise to the group invariant solution

$$
u=\frac{2}{\alpha}\left(\frac{E(z)}{\sqrt{t}}-1\right), \quad v=\frac{F(z)}{t},
$$

where $z=\frac{x}{\sqrt{t}}+\frac{2}{\alpha} \sqrt{t}$ is an invariant of the symmetry $\alpha X_{1}+X_{3}$. Substitution of (2) into (1) results in the system of ordinary differential equations (ODEs) where $E$ and $F$ satisfy

$$
\begin{aligned}
& -\frac{\alpha}{2} F^{\prime} z-\alpha F+2 F E^{\prime}+2 E F^{\prime}+2 E^{\prime \prime \prime}=0, \\
& -\frac{1}{\alpha}\left(\frac{E^{\prime}}{z}+E\right)+F^{\prime}+\frac{4}{\alpha^{2}} E E^{\prime}=0 . \\
& \text { Case } 2 . c_{1}=c_{2}=c_{4}=0, c_{3}=1 .
\end{aligned}
$$

The symmetry $X_{3}$ gives rise to the group-invariant solution of the form

$$
F^{\prime}+\frac{1}{z} F=0, \quad E^{\prime}+\frac{1}{z} E=0 .
$$

Solving the above system of ODEs one obtains the rational solution

$$
u(x, t)=\frac{c_{1}}{t}+\frac{x}{t}, \quad v(x, t)=\frac{c_{2}}{t} .
$$

Case 3. $c_{1}=a_{1}, c_{2}=c_{3}=0, c_{4}=1$.

By solving the corresponding Lagrange system for the symmetry $a_{1} X_{1}+X_{4}$, one obtains an invariant $z=$ $x^{2}\left(a_{1}-t\right)^{-1}$ and the group-invariant solution of the form

$$
u(x, t)=\frac{E(z)}{\sqrt{a_{1}-t}}, \quad v(x, t)=\frac{F(z)}{a_{1}-t},
$$

where the functions $E$ and $F$ satisfy the following system of ODEs:

$$
\begin{aligned}
& 8 z^{\frac{3}{2}} E^{\prime \prime \prime}+12 z^{\frac{1}{2}} E^{\prime \prime}+2 z^{\frac{1}{2}}\left(E^{\prime} F+E F^{\prime}\right)+z F^{\prime}+F=0, \\
& 2 z E^{\prime}+4 z^{\frac{1}{2}} F^{\prime}+4 z^{\frac{1}{2}} E E^{\prime}+E=0 .
\end{aligned}
$$

Case 4. $c_{1}=c_{3}=0, c_{2}=1, c_{4}=c$.

In this case by solving the sorresponding Lagrange system for the symmetry $X_{2}+c X_{4}$, one obtains an invariant $z=x-c t$ and the group invariant solution of the form

$$
u=u(z), \quad v=v(z)
$$

and proceeding as before we find

$$
u^{\prime \prime \prime}+u v^{\prime}+u^{\prime} v-c v^{\prime}=0, \quad u u^{\prime}-c u^{\prime}+v^{\prime}=0,
$$

where after integrating (6) and substituting $v=c u-\frac{u^{2}}{2}$, we find

$$
u^{\prime \prime}-\frac{u^{3}}{2}+\frac{3}{2} c u^{2}-c^{2} u=0 .
$$

\subsection{Exact solutions using simplest equation method}

The simplest equation method, which was introduced by Kudryashov [28-30] will be used for obtaining the exact solutions of evolution type systems. The simplest equation that will be used is the Ricatti equation

$$
G^{\prime}(z)=b G(z)+d G(z)^{2},
$$

where $b$ and $d$ are arbitrary constants. This equation is a well-known nonlinear ordinary differential equation which possesses exact solutions given by elementary functions. The solutions can be expressed as

$$
G(z)=\frac{b \exp \left(b\left(z+z_{0}\right)\right)}{1-d \exp \left(b\left(z+z_{0}\right)\right)},
$$

for the case when $d<0, b>0$, and

$$
G(z)=-\frac{b \exp \left(b\left(z+z_{0}\right)\right)}{1+d \exp \left(b\left(z+z_{0}\right)\right)},
$$

for $d>0, b<0$. Here $z_{0}$ is a constant of integration.

Let us consider the solution of (7) of the form

$$
u(z)=\sum_{i=0}^{M} A_{i}(G(z))^{i},
$$

where $G(z)$ satisfies the Riccati equation (8), $M$ is a positive integer that can be determined by balancing procedure, and $A_{0}, A_{1}, \ldots, A_{M}$ are parameters to be determined.

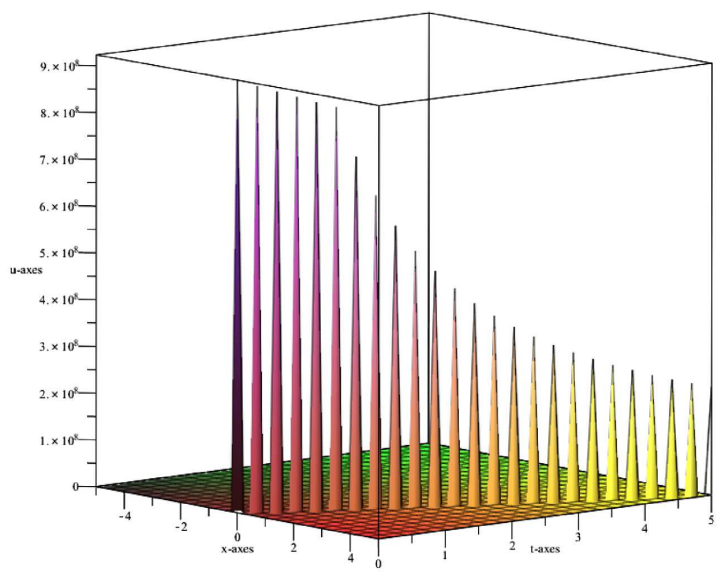

Fig. 1. Evolution of travelling wave solution (14) with parameters $C=1, z_{0}=0, b=3, d=1$.

The balancing procedure yields $M=1$, so the solution of (7) is of form

$$
u(z)=A_{0}+A_{1} G(z) .
$$

Substituting (12) into (7) and making use of the Ricatti equation (8) and the equating all coefficients of the functions $G^{i}$ to zero, we obtain an algebraic system of equations in terms of $A_{0}$ and $A_{1}$. Solving these algebraic equations, with the aid of Maple, we obtain the following value of $A_{0}$ and $A_{1}$ :

$$
A_{0}=2 c, A_{1}=\frac{2 b d}{c}, b= \pm c, c \neq 0 .
$$

Therefore, when $d<0, b>0$ the solution of (7) and hence the solutions of (1) are given by (see Fig. 1): 


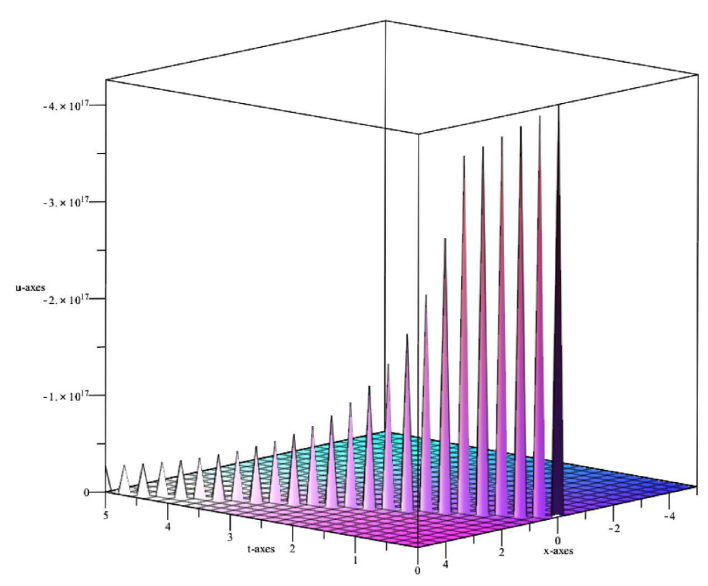

Fig. 2. Evolution of travelling wave solution (15) with parameters $C=-1, z_{0}=0, b=2, d=-1$.

$$
\begin{aligned}
& u(x, t)=2 c+\frac{2 b^{2} d \exp \left(b\left(x-c t+z_{0}\right)\right)}{c\left(1-d \exp \left(b\left(x-c t+z_{0}\right)\right)\right)} \\
& v(x, t)=\left[-2\left(c^{2} \exp \left(b\left(-x+c t-z_{0}\right)\right)-c^{2} d+b^{2} d\right)\right. \\
& \left.\quad \times b^{2} d \exp \left(-2 b\left(-x+c t-z_{0}\right)\right)\right] \\
& /\left[c^{2}\left(d \exp \left(-b\left(-x+c t-z_{0}\right)\right)-1\right)^{2}\right] .
\end{aligned}
$$

When $d>0, b<0$ the solution of (7) and hence the solutions of (1) are given by (see Fig. 2):

$$
\begin{aligned}
& u(x, t)=2 c-\frac{2 b^{2} d \exp \left(b\left(x-c t+z_{0}\right)\right)}{c\left(1+d \exp \left(b\left(x-c t+z_{0}\right)\right)\right)} \\
& v(x, t)=\left[-2\left(-c^{2} \exp \left(b\left(-x+c t-z_{0}\right)\right)-c^{2} d+b^{2} d\right)\right. \\
& \left.\quad \times b^{2} d \exp \left(-2 b\left(-x+c t-z_{0}\right)\right)\right] \\
& /\left[c^{2}\left(d \exp \left(-b\left(-x+c t-z_{0}\right)\right)+1\right)^{2}\right] .
\end{aligned}
$$

\section{Conservation laws}

Conservation laws plays an important role in the analysis of basic properties of solutions. The existence of a large number of conservation laws of a partial differential equation (PDE) is a strong indication of its integrability [14]. They are used for analysis, in particular, existence, uniqueness, and stability analysis and construction of numerical schemes [18]. In addition, in the numerical integration of PDEs [31, 32], for example, to control numerical errors, conservation laws are also used.

In this section, we construct conservation laws for (1). For the details see e.g., [14, 18, 19, 22].

Consider a $k$-th order system of partial differential equations (PDEs) of $n$ independent variables $x=\left(x^{1}, x^{2}, \ldots, x^{n}\right)$ and $m$ dependent variables $u=$ $\left(u^{1}, u^{2}, \ldots, u^{n}\right)$, namely

$$
E_{\alpha}\left(x, u, u_{(1)}, \ldots, u_{(k)}\right)=0, \quad \alpha=1, \ldots, m,
$$

where $u_{(1)}, u_{(2)}, \ldots, u_{(k)}$ denote the collections of all first, second,..., $k$-th order partial derivatives, i.e., $u_{i}^{\alpha}=$
$D_{i}\left(u^{\alpha}\right), u_{i j}^{\alpha}=D_{j} D_{i}\left(u^{\alpha}\right), \ldots$, respectively, where the total derivative operator with respect to $x^{i}$ is given by

$$
D_{i}=\frac{\partial}{\partial x^{i}}+u_{i}^{\alpha} \frac{\partial}{\partial u^{\alpha}}+u_{i j}^{\alpha} \frac{\partial}{\partial u_{j}^{\alpha}}+\ldots, \quad j=1, \ldots, n
$$

where the summation convertion is used whenever appropriate. The $n$-tuple vector

$$
T=\left(T^{1}, T^{2}, \ldots, T^{n}\right), \quad T^{j} \in A, \quad j=1, \ldots, n,
$$

is a conserved vector of (16) if $T^{i}$ satisfied

$$
\left.D_{i} T^{i}\right|_{(16)}=0 .
$$

The equation (18) is called a local conservation law of system (16).

It can be shown that every admitted conservation laws arises from multipliers $Q^{\alpha}\left(x, u, u_{(1)}, \ldots\right)$ such that

$$
Q^{\alpha} E_{\alpha}=D_{i} T^{i}
$$

holds identically. In the multiplier approach for conservation laws, one takes the variational derivative of (19) that is,

$$
\frac{\delta}{\delta u^{\beta}}\left(Q^{\alpha} E_{\alpha}\right)=0
$$

holds for arbitrary functions of $u\left(x^{1}, x^{2}, \ldots, x^{n}\right)$. Here we will consider multipliers of the second order $\Lambda_{\alpha}=$ $\Lambda_{\alpha}\left(x, t, u, v, u_{x}, v_{x}, u_{x x}, v_{x x}\right)$. Once the multipliers are obtained the conserved vectors are calculated via a homotopy formula ([22] and [24]).

\subsection{Conservation laws of (1)}

For the variant Boussinesq system (1), we see that the two second order multipliers (with the aid of GeM [18], see also [23, 24]), namely $\Lambda_{1}\left(x, t, u, v, u_{x}, v_{x}, u_{x x}, v_{x x}\right)$, $\Lambda_{2}\left(x, t, u, v, u_{x}, v_{x}, u_{x x}, v_{x x}\right)$ are given by

$$
\begin{aligned}
\Lambda_{1} & =\frac{1}{6} c_{2} u^{3}+\frac{1}{2} c_{3} u^{2}+\frac{1}{6}\left(6 c_{1} t+6 c_{2} v+6 c_{4}\right) u \\
- & c_{1} x+\frac{2}{3} c_{2} u_{x x}+c_{3} v+c_{6}, \\
\Lambda_{2} & =\frac{1}{6}\left(6 u u_{x x}+3 u_{x}^{2}+4 v_{x x}+3 u^{2} v+3 v^{2}\right) c_{2} \\
& +\frac{1}{6}\left(6 c_{1} t+6 c_{4}+6 c_{3} u\right) v+c_{5}+c_{3} u_{x x},
\end{aligned}
$$

where $C_{i}, i=1,2,3,4,5,6$ are arbitrary constants.

Corresponding to the above multipliers we have the following six conserved vectors of (1):

$C_{1}^{t}=t u v-x v$,

$C_{1}^{x}=-\frac{1}{2} t u_{x}^{2}+u_{x}+\frac{1}{2} t v^{2}+t u^{2} v-x u v+t u u_{x x}-x u_{x x} ;$

$C_{2}^{t}=\frac{1}{6} v u^{3}+\frac{1}{2} v^{2} u+\frac{1}{3} u^{2} u_{x x}+\frac{1}{6} u u_{x}^{2}+\frac{1}{3} v u_{x x}+\frac{1}{3} u v_{x x}$,

$C_{2}^{x}=\frac{1}{3} v_{x} u_{t}+\frac{1}{3} v_{x}^{2}-\frac{1}{6} v u_{x}^{2}+\frac{1}{3} u_{x x}^{2}+\frac{1}{3} u_{x} v_{t}-\frac{1}{3} v u_{t x}$

$-\frac{1}{3} u v_{t x}+\frac{1}{4} u^{2} u_{x}^{2}+\frac{1}{6} u^{4} v+\frac{1}{6} u_{x x} u^{3}+\frac{1}{3} u u_{x} u_{t}$

$+\frac{1}{6} v^{3}-\frac{1}{3} u^{2} u_{t x}+\frac{3}{4} u^{2} v^{2}+\frac{2}{3} v_{x} u u_{x}+v u u_{x x} ;$ 


$$
\begin{aligned}
& C_{3}^{t}=\frac{1}{2} v u^{2}+\frac{1}{2} v^{2}+\frac{1}{2} u u_{x x}, \\
& C_{3}^{x}=\frac{1}{2} u^{2} u_{x x}+v u_{x x}+\frac{1}{2} u_{x} u_{t}+\frac{1}{2} v u^{3}+v^{2} u-\frac{1}{2} u u_{t x} ; \\
& C_{4}^{t}=u v, \quad C_{4}^{x}=-\frac{1}{2} u_{x}^{2}+\frac{1}{2} v^{2}+u u_{x x}+v u^{2} ; \\
& C_{5}^{t}=u, \quad C_{5}^{x}=\frac{1}{2} u^{2}+v ; \quad C_{6}^{t}=v, \quad C_{6}^{x}=v u+u_{x x} .
\end{aligned}
$$

\section{Concluding remarks}

In this paper, we have constructed exact solutions and conservation laws for third order variant Boussinesq system. We used the Lie symmetry method to derive all the Lie point symmetry generators admitted by the system (1).

Then the symmetry generators of (1) have been used to construct symmetry reductions and exact travelling wave solutions with the aid of simplest equation method. As stated in [24], the obtained exact solutions can be used as benchmarks against the numerical simulations. Moreover, we constructed conservation laws for the system (1) via the multiplier approach. In the multiplier approach to construct conservation laws for (1), the second order multipliers are considered. For the system (1), this method gave rise to six multipliers and thus six conserved vectors were obtained.

The conserved vectors obtained here can be used in reductions and solutions of the underlying system [33]. In future work, with the aid of conservation laws of the system, nonlocal symmetries such as potential and nonclassical potential symmetries will be obtained. These symmetries enable one to obtain new interesting solutions of the considered system.

\section{References}

[1] V.O. Vakhnenko, E.J. Parkes, A.J. Morrison, Chaos Solitons Fract. 17, 683 (2003).

[2] R. Hirota, Phys. Rev. Lett. 27, 1192 (1971).

[3] Y. Ma, X. Geng, Appl. Math. Comput. 218, 6963 (2012).

[4] A. Zerarka, S. Ouamane, S. Attaf, Appl. Math. Comput. 217, 2897 (2010).

[5] M.L. Wang, Y.B. Zhou, Z.B. Li, Phys. Lett. A 216 , 67 (1996)

[6] A. Bekir, Commun. Nonlin. Sci. Numer. Simulat. 13, 1748 (2008).

[7] A. Bekir, Phys. Lett. A 372, 3400 (2008).

[8] E. Fan, Y.C. Hon, Chaos Solitons Fract. 15, 559 (2003).

[9] B. Muatjetjeja, C.M. Khalique, Abstract Appl. Anal. 2014, 169694 (2014).
[10] M. Wang, Phys. Lett. A 199, 169 (1995).

[11] R. Naz, F.M. Mahomed, T. Hayat, Appl. Math. Lett. 23, 883 (2010).

[12] I.M. Anderson, M.E. Fels, C.G. Torre, Commun. Math. Phys. 212, 653 (2000).

[13] G.W. Bluman, S.C. Anco, Symmetry and Integration Methods for Differential Equations, Vol. 154 of Applied Mathematical Sciences, Springer, New York 2002.

[14] P.J. Olver, Application of Lie Groups to Differential Equations, Springer-Verlag, New York 1993.

[15] N.H. Ibragimov, CRC Handbook of Lie Group Analysis of Differential Equations, Vol. 1, Symmetries, Exact Solutions and Conservation Laws, CRC Press, Boca Raton, Florida 1995.

[16] C. Rogers, W. Shadwick, Nonlinear Boundary Value Problems in Science and Engineering. Mathematics in Science and Enginering, Vol. 183, Ed. W.F. Ames, Academic Press, Boston 1989.

[17] N.H. Ibragimov, R. Khamitova, B. Thide, J. Math. Phys. 48, 053523 (2007).

[18] A.F. Cheviakov, Comput. Phys. Commun. 176, 48 (2007).

[19] H. Steudel, Z. Naturforsch. 17A, 129 (1962).

[20] A.H. Kara, F.M. Mahomed, Int. J. Theor. Phys. 39, 23 (2000).

[21] A.H. Kara, F.M. Mahomed, Nonlinear Dynam. 45, 367 (2006).

[22] S.C. Anco, G.W. Bluman, Eur. J. Appl. Math. 13, $545(2002)$.

[23] A.R. Adem, C.M. Khalique, Comput. Fluids 81, 10 (2013).

[24] A.R. Adem, C.M. Khalique, Commun. Nonlin. Sci. Numer. Simulat. 17, 3465 (2012).

[25] H. Triki, A.H. Kara, A.H. Bhrawy, A. Biswas, Acta Phys. Pol. A 125, 1099 (2014).

[26] I.E. Mhlanga, C.M. Khalique, J. Appl. Math. 2012, 389017 (2012).

[27] N.H. Ibragimov, J. Math. Anal. Appl. 333, 311 (2007).

[28] N.A. Kudryashov, Chaos Solitons Fract. 24, 1217 (2005).

[29] N.A. Kudryashov, Phys. Lett. A 342, 99 (2005).

[30] H. Jafari, N. Kadkhoda, C.M. Kahlique, Abstract Appl. Anal. 2012, 350287 (2012).

[31] R.J. LeVeque, Numerical Methods for Conservation Laws, Lectures in Mathematics, Birkhauser Verlag, Basel 1992.

[32] T. Wolf, Europ. J. Appl. Math. 2, 129 (2002).

[33] A.H. Bokhari, A.Y. Al-Dweik, A.H. Kara, F.M. Mahomed, F.D. Zaman, Commun. Nonlin. Sci. Numer. Simulat. 16, 1244 (2011). 